\title{
Uma Análise dos Valores Pessoais de Profissionais de Tecnologia da Informação
}

\author{
Lucimara Amorim da $\operatorname{Costa}^{1}$, Edson Luiz Pereira ${ }^{2}$, Enock Godoy de Souza ${ }^{3}$ \\ ${ }^{1,2}$ Mestrandos em Administração na Universidade Presbiteriana Mackenzie \\ ${ }^{3}$ Professor da Faculdade de Informática e Administração Paulista (FIAP) e Mestre em \\ Sistemas de Informação pela London School of Economics and Political Science (LSE) \\ lucimara.amorim@uol.com.br, edsonlp1@hotmail.com, enock.godoy@acm.org
}

\begin{abstract}
The objective of this article is presenting an analysis of the personal values of a sample of Information Technology professionals, aiming to understand the values' hierarchy and its correlations, using the motivational types proposed by Schwartz as a basis. The main results demonstrate that the individuals prioritize characteristics of conservation and self-transcendence, aiming aspects like self-determination and estimulation, valuing intelligence, capability, freedom and independence.
\end{abstract}

Resumo: O objetivo deste artigo é apresentar uma análise dos valores pessoais de uma amostra de profissionais que atuam na área de Tecnologia da Informação, buscando compreender a hierarquia dos valores e a relação entre eles, usando como base os tipos motivacionais proposto por Schwartz. Os principais resultados o trabalho demonstram que os indivíduos priorizam aspectos de conservação e autotranscendência, buscam auto-realização através da auto-determinação e estimulação, valorizando inteligência, capacidade, liberdade e independência.

\section{Introdução}

O estudo de valores tem despertado interesse no desenvolvimento de pesquisas pois são considerados a parte central da cultura (Hofstede, 2001), influenciando de forma decisiva a dinâmica das relações entre os indivíduos e as sociedades.

Desde os experimentos de Hawthorne, realizados por Elton Mayo, as empresas começaram a perceber a importância das pessoas na dinâmica das organizações e como seu humor e sentimento em relação à empresa poderiam contribuir para que os produtos tivessem uma melhor qualidade. Acompanhando a evolução das organizações desde a Revolução Industrial, podemos perceber uma evolução do foco no processo e na linha de produção para um interesse maior nas pessoas que fazem parte desse processo, afetando o "modus operandi" das organizações, que passaram a entender que as pessoas não são máquinas que podem ser controladas e previstas totalmente.

$\mathrm{O}$ estudo de valores apresenta aspectos interessantes que contribuem na tentativa de compreender como determinados indivíduos pensam, pois os valores que as pessoas possuem contribuem de maneira relevante nos processos de tomada de decisão utilizados durante a vida. Analogamente ao computador, os valores agem como um "programa instalado" durante a primeira infância através da socialização. Dentre as pesquisas relacionadas a valores, algumas declaram que a pessoa vai naturalmente reagir com base nesse "programa", enquanto outras querem entender como se faz a mudança dos valores e quais permitem a possibilidade de ser mudados.

Este trabalho busca diagnosticar a percepção dos indivíduos sobre valores numa amostra de profissionais da área de Tecnologia da Informação (TI), analisando seus 
valores pessoais, utilizando para isso uma escala já desenvolvida (Schwartz, 1992) e validada no Brasil (Tamayo e Schwartz, 1993).

A necessidade de intensificarmos a quantidade e qualidade das pesquisas sobre o ambiente social brasileiro é aderente às idéias de De Souza e Fontana (2004), que utilizando a abordagem da Escola Sistêmica de Estratégia (Whittington, 2001), de que as estratégias devem ser adaptadas ao contexto social em que se encontram, recomendam o desenvolvimento de mais pesquisas sobre o contexto sociológico brasileiro, com o intuito de expandir o conhecimento sobre como esses conceitos são aplicados em nosso país. A essa recomendação se soma a percepção de uma grande carência de estudos realizados sobre nossa cultura, proporcionando a esta pesquisa a oportunidade de contribuir para a evolução deste entendimento.

\section{O Estudo dos Valores}

O estudo de valores passou a ter maior dimensão a partir da década de 70 , quando passou a ser mais enfatizada a importância da prioridade de valores dos indivíduos para se entender e prever decisões de comportamento e atitude. Rockeach (1973) diz que os valores são crenças transituacionais que se encontram hierarquicamente organizadas e servem de critério para o comportamento (apud Ros, 2001). A maior contribuição do trabalho de Rokeach (1973) é a percepção de que os valores estão organizados em hierarquia de importância e assim ele divide os valores em dois tipos: (1) instrumentais, são os valores que representam os meios para alcançar os fins da existência humana, por exemplo: honestidade, obediência, capacidade, responsabilidade e (2) terminais, são aqueles que respondem pelas necessidades da existência humana, por exemplo: segurança familiar, liberdade, segurança nacional, reconhecimento social. (Rokeach, 1973, apud Ros, 2001). Ele também desenvolve um instrumento para medir os valores, o RVS (Rokeach Value Survey), que consta de 18 valores terminais e 18 valores instrumentais, e a criação do método de autoconfrontação dos valores, que consiste na solicitação de que o indivíduo organize os valores em ordem de importância para si.

Para Rohan (2000), valor é um princípio implícito e analógico construído através de julgamentos sobre a capacidade das coisas, pessoas, ações e atividades que habilitam a melhor forma de viver. Ela propõe um processo onde as prioridades de valores coordenam as decisões de atitude e comportamento dos indivíduos.

Schwartz (2001) define valores como crenças que traduzem formas de comportamento desejadas ou desejáveis, que transcendem situações específicas e orientam a escolha e/ou avaliação de comportamentos e pessoas, além disso, valores são ordenados por importância relativa a outros valores, ou ainda sistema de prioridades de valores.

$\mathrm{Na}$ Psicologia Social, o próprio conceito de valores salienta a sua dimensão motivacional. Eles são definidos como princípios transituacionais, organizados hierarquicamente, relativos a estados de existência ou modelos de comportamentos desejáveis, que orientam a vida do indivíduo e expressam interesses individuais, coletivos ou mistos, bem como diversos tipos motivacionais. Esta definição implica que os valores são metas que o indivíduo fixa a si próprio, relativas a estados de existência (valores terminais) ou a modelos de comportamento desejáveis (valores instrumentais) (Rokeach, 1973, apud Tamayo e Schwartz, 1993).

Vários autores consideram fonte dos valores as exigências universais do ser humano (Kluckhon, 1951; Rokeach, 1973, Schwartz e Bilsky, 1987, apud Tamayo e Schwartz, 1993). Essas exigências pré-existem ao indivíduo e são constituídas por: (1) 
necessidades biológicas do organismo, (2) necessidades sociais relativas à regulação das interações interpessoais e (3) necessidades sócio-institucionais referentes à sobrevivência e bem-estar dos grupos.

Schwartz e Bilsky (1987, apud Tamayo e Schwartz, 1993) consideram que as exigências universais do ser humano constituem a fonte dos valores que se expressam através de tipos motivacionais. Nesse trabalho usaremos essa abordagem para estudar os valores pessoais de profissionais de TI.

\section{Valores Pessoais}

Schwartz (1992) desenvolveu uma tipologia sobre o conteúdo dos valores humanos baseando-se nas metas motivacionais que eles expressam.

A estrutura dos valores humanos está formada por 10 tipos motivacionais: (1) Hedonismo: Prazer e gratificação sensual para si mesmo, geralmente associado a valores socialmente reconhecidos. (2) Auto-realização: É o sucesso pessoal obtido através de uma demonstração de competência. (3) Poder social: Busca de status social, prestígio e controle sobre pessoas e recursos. (4) Auto-determinação: Independência de pensamento, ação e opção. (5) Conformidade: Controle de impulsos e ações que podem violar normas sociais ou prejudicar os outros. (6) Benevolência: Interesse e preocupação com o bem-estar de pessoas íntimas. (7) Segurança: Integridade pessoal, estabilidade da sociedade, do relacionamento e de si mesmo. (8) Tradição: Respeito e aceitação dos ideais e costumes da sociedade. (9) Estimulação: Procura de excitação, novidade e mudança, desafio. (10) Filantropia ou Universalismo: Tolerância, compreensão e promoção do bem-estar de todos e da natureza.

Esses 10 tipos motivacionais formam uma estrutura de relações de compatibilidade e contradição entre eles, refletindo num agrupamento em duas dimensões bipolares: (1) Autopromoção versus Autotranscendência e (2) Abertura à Mudança versus Conservação.

Além da ordenação nas duas dimensões, os tipos motivacionais também expressam interesses individuais (auto-determinação, estimulação, hedonismo, autorealização e poder), interesses coletivos (benevolência, tradição e conformidade) e mistos (filantropia e segurança) (Tamayo e Schwartz, 1993).

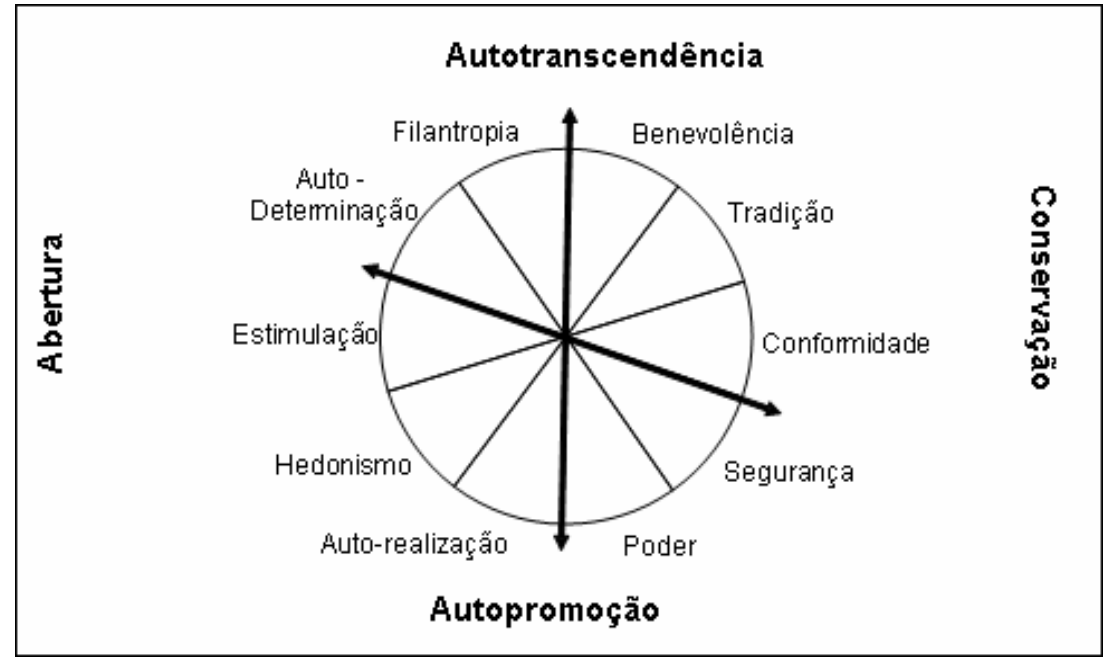

Figura 1. Tipos Motivacionais dos Valores Pessoais Fonte: adaptado de Schwartz 2001 


\section{Pesquisa sobre motivação dos profissionais de TI}

Uma das pesquisas mais citadas na área de TI é a realizada por Couger e Zawacki (1980). A pesquisa é baseada nas seguintes premissas ligadas a definição de motivação: (1) O comportamento humano é causado (possui uma causa), (2) $O$ comportamento humano é orientado por objetivos e (3) O comportamento humano é motivado. (Leavitt 1964, apud Couger e Zawacki, 1980). A partir disso, Couger e Zawacki (1980) publicaram uma análise dos fatores motivacionais dos profissionais de TI, cuja pesquisa incluiu 2500 questionários, de 50 organizações. Atualmente sua base de dados possui 18000 questionários respondidos por norte-americanos e 19500 pessoas de outros países (McNurlin e Sprague, 2002).

Dentre as conclusões que podem ser extraídas da pesquisa, os dados coletados nessa pesquisa demonstraram que os profissionais de desenvolvimento de sistemas possuem uma alta necessidade de aprimoramento, essa necessidade de aprimoramento sugere que, para manter esse profissional com alto grau de motivação, é necessário investir na sua evolução, ou seja, esses profissionais precisam ser sempre desafiados a trabalhar com a última tecnologia e o seu crescimento profissional deve ser incentivado. Outro resultado dessa pesquisa foi a constatação da baixa necessidade de interação pessoal dos analistas e programadores. A baixa necessidade de interação social não fica restrita, no entanto, a analistas e programadores. As pesquisas também constataram que esses profissionais anseiam por um melhor retorno (feedback) de seus superiores, que também possuem uma baixa necessidade de interação pessoal. (Couger e Zawacki, 1980; McNurlin e Sprangue 2002)

\section{Procedimentos metodológicos}

A pesquisa apresentada nesse trabalho busca analisar os valores pessoais dos indivíduos de uma amostra de profissionais de TI. Entender a maneira como esses indivíduos percebem o mundo e como seus valores podem ser determinantes na maneira como os mesmos se relacionam e tomam decisões.

\section{Técnica de coleta dos dados}

Os questionários foram administrados através de um site na Internet, onde os respondentes acessavam o link informado e se deparavam com o questionário e as respectivas instruções onde eles deveriam escolher para cada questão a resposta que mais se adequasse, não podendo deixar nenhuma questão sem resposta.

Foi aplicada a escala de importância dos valores pessoais.

\section{Escala dos valores pessoais}

Foi utilizada a escala de Tamayo e Schwartz (1993). Ela consta de 56 valores, sendo 30 terminais e 26 instrumentais, da escala original dos 10 tipos motivacionais de Schwartz (1992) desenvolvida na pesquisa intercultural, sendo introduzidos mais quatro valores, que parecem ser peculiares à cultura brasileira (Tamayo e Schwartz, 1993). A importância dos valores "como princípios que orientam a minha vida" foi avaliada através de uma escala de 0 a 6 . Quanto mais alto o número, mais importante é o valor para a pessoa.

\section{Técnica de análise e tratamento dos dados}

Para análise dos dados, utilizou-se o programa estatístico SPSS (Statistical Package of Social Science). 
Para interpretação dos dados verificou-se principalmente a hierarquia dos valores e as correlações entre os valores pessoais, procurando identificar os aspectos que mais se destacassem para entender o perfil desenhado pelos profissionais de tecnologia pesquisados.

\section{A empresa pesquisada}

A pesquisa foi realizada com funcionários da região da Grande São Paulo, de uma empresa multinacional americana do setor de TI, com filiais nas principais capitais brasileiras e mais de cinco mil funcionários no Brasil.

As atividades da EMPRESA se estendem hoje por mais de 150 países. As fábricas e laboratórios funcionam em 15 diferentes países.

A EMPRESA se propõe a ser a melhor empresa de serviços de TI. Sua mais alta prioridade é usar toda a potencialidade da sua tecnologia para oferecer produtos cada vez melhores, mais rápidos, mais baratos e mais fáceis de usar.

Todos os dados sobre a empresa descritos nesse trabalho foram obtidos através de informações disponíveis na Internet, e o nome da empresa foi preservado substituindo-o por "EMPRESA".

\section{Os sujeitos da pesquisa}

A escolha dos sujeitos da pesquisa foi proposital, em função das condições de acesso, disponibilidade dos participantes e interesse da organização.

O perfil dos 291 participantes dessa pesquisa é apresentado a partir dos seguintes aspectos: gênero, idade, tempo de trabalho na empresa, cargo e departamento:

\section{Tabela 1 - Perfil dos sujeitos}

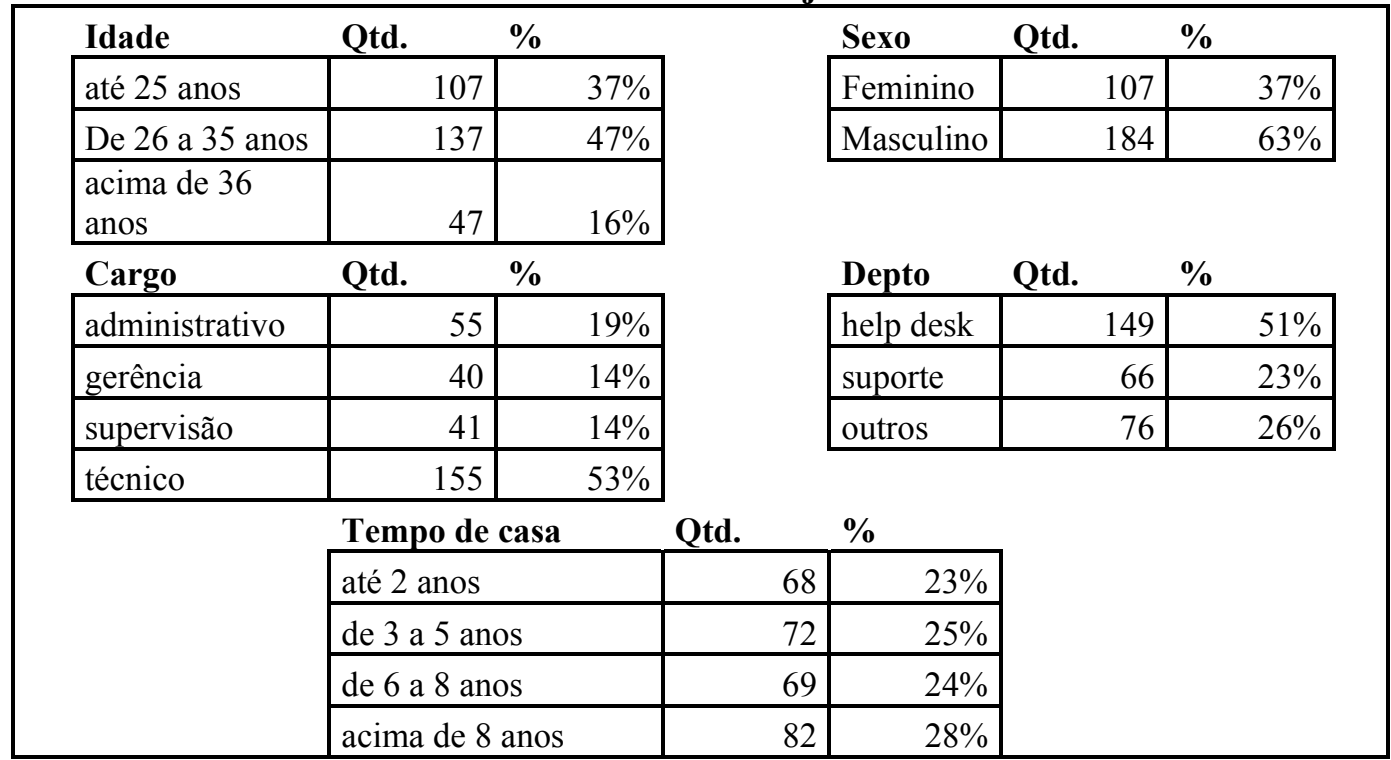

\section{Apresentação e Análise dos Resultados}

A análise dos resultados será dividida em duas etapas: (1) análise da hierarquia dos valores pessoais e (2) análise das correlações.

Serão levados em conta os fatores que mais se destacarem tanto na hierarquia quanto na análise de correlações, pois considera-se que esses são os que poderão trazer informações mais relevantes sobre a dinâmica do grupo estudado.

\section{Hierarquia dos valores pessoais}


A hierarquia dos valores se faz importante pois é possível perceber as preferências pessoais do grupo através dela, e com isso identificar quais os anseios desse tipo de profissional, seus principais interesses e motivações.

$\mathrm{Na}$ hierarquia dos valores pessoais dos profissionais de TI dessa amostra, percebemos que os valores preferidos do grupo estão mais relacionados às dimensões de conservação e autotranscendência.

O nível mais alto na hierarquia é comporto pelos valores: Segurança familiar (proteção para minha família), Honesto (ser sincero, autêntico), Trabalho (modo digno de ganhar a vida), Harmonia interior (em paz comigo mesmo). Responsável (ser fidedigno, confiável), Capaz (ser competente, eficaz, eficiente), Leal (ser fiel aos amigos e grupos).

O nível mais baixo na hierarquia foi constituído pelos valores: Poder social (controle sobre os outros, domínio), Autoridade (direito de liderar ou de mandar), Respeito pela tradição (preservação de costumes vigentes há longo tempo) e Devoto (apegar-me fortemente à fé religiosa), esses valores encontram-se nas dimensões de autopromoção e conservação, e nessa última especificamente no tipo de tradição, pois outros tipos de valores da dimensão de conservação são muito valorizados pelos indivíduos.

\section{Correlação dos valores pessoais}

O objetivo do estudo correlacional é a determinação da força do relacionamento entre duas observações emparelhadas. O termo "correlação" significa literalmente "corelacionamento", pois indica até que ponto os valores de uma variável estão relacionados com os de outra (Stevenson 1981).

A Tabela 2 apresenta as correlações entre as dimensões, com base nelas, foram também analisadas as correlações entre os tipos motivacionais que mais se destacaram, a tabela com essas correlações não pôde ser disponibilizada nesse artigo por limitação de espaço.

Tabela 2- Correlação entre as dimensões

\begin{tabular}{|c|c|c|c|c|}
\hline \multicolumn{5}{|c|}{$\begin{array}{c}\text { Correlação dos Valores Pessoais } \\
\text { (Dimensões) }\end{array}$} \\
\hline Pessoais $\downarrow$ Pessoais $\rightarrow$ & $\begin{array}{l}\text { Abertura à } \\
\text { mudança }\end{array}$ & \begin{tabular}{|l} 
Auto \\
Promoção
\end{tabular} & $\begin{array}{l}\text { Auto } \\
\text { Transcendência } \\
\end{array}$ & Conservação \\
\hline Abertura à mudança & 1 &, $795\left(^{* *}\right)$ &, $700\left(^{\star \star *}\right)$ &, $657\left({ }^{* \star}\right)$ \\
\hline Auto Promoção &, $795\left(^{* *}\right)$ & 1 &, $611\left(^{* *}\right)$ &, $657\left({ }^{* *}\right)$ \\
\hline Auto Transcendência &, $700\left(^{* *}\right)$ &, $611\left({ }^{* *}\right)$ & 1 &, $837\left({ }^{* \star}\right)$ \\
\hline Conservação &, $657\left(^{* \star}\right)$ &, $657\left(^{* *}\right)$ &, $837\left(^{* *}\right)$ & 1 \\
\hline
\end{tabular}

$\mathrm{Na}$ correlação das dimensões percebemos naturalmente que todas são consideradas altas ou fortes pois estamos analisando aspectos dos valores pessoais entre si, quando essa análise é feita contra outros tipos de valores, por exemplo, valores do trabalho (Porto e Tamayo, 2003) ou valores organizacionais (Oliveira e Tamayo, 2004) os dados podem apresentar correlações mais fracas ou até negativas. 
Para os profissionais de tecnologia pesquisados percebemos que a correlação mais forte está entre as dimensões de autotranscendência (benevolência e filantropia) e conservação (conformidade, segurança e tradição), é interessante percebermos que essas duas dimensões também são as que aparecem como as mais valorizadas conforme a hierarquia dos valores pessoais dos indivíduos, ou seja além de elas serem muito valorizadas por eles os valores que compõem essas dimensões são considerados pelos indivíduos como possuindo uma forte relação entre si.

Os valores que se destacam nessas dimensões e que possuem as mais fortes correlações nos tipos motivacionais são os de conformidade e benevolência que tem como exemplos: ser honesto, ser responsável, ser leal, amizade verdadeira, trabalho, respeito pelos mais velhos, polidez, ser prestativo, ter harmonia interior, sabedoria, assim podemos perceber que esses indivíduos demonstram características que poderiam classificá-los como confiáveis e que essas características também são favoráveis ao desenvolvimento de trabalho em equipe.

A segunda correlação mais destacada foi a correlação entre as dimensões de abertura à mudança (auto-determinação e estimulação) e autopromoção (hedonismo, auto-realização e poder social). Nessa correlação destacam-se os tipos motivacionais de auto-realização, estimulação e auto-determinação que são compostos pelos seguintes valores: ser capaz, ser inteligente, bem sucedido, audacioso, liberdade, independente, privacidade, criatividade, curioso. Esses indivíduos tendem a buscar sua auto-realização através da auto-determinação e estimulação, valorizam a inteligência e a capacidade, gostam de ter liberdade e independência.

\section{Conclusão}

Através da análise dos valores pessoais podemos estabelecer relações que contribuem para o entendimento de um grupo de pessoas e como eles se relacionam, $o$ que valorizam, podemos ter indicações de como esses indivíduos se comportam e o que poderia ser mais adequado para motivá-los.

Algumas constatações desta pesquisa podem ser comparadas à pesquisa de Couger e Zawacki (1980) sobre os fatores motivacionais dos profissionais de TI, onde os autores concluíram que os profissionais de desenvolvimento de sistemas possuem uma alta necessidade de aprimoramento, no caso desta pesquisa realizada com indivíduos predominantemente da área de suporte técnico, percebeu-se a valorização da capacidade, inteligência e importância do trabalho demonstrados através dos valores pessoais dos indivíduos indicando assim um alinhamento dos resultados das duas pesquisas. Além disso, os indivíduos desta pesquisa buscam sua auto-realização através da auto-determinação e estimulação.

Em oposição, encontramos na pesquisa de Couger e Zawacki (1980) a constatação de uma baixa necessidade de interação pessoal e nesta amostra percebemos que valores como a amizade verdadeira, o respeito pelos outros e lealdadesão muito valorizados pelos indivíduos, cabendo neste caso uma investigação mais profunda para entender se o ambiente ou o tipo de valor poderiam influenciar esse resultado. Uma sugestão seria estender a pesquisa com valores do trabalho e valores organizacionais.

Devemos ressaltar que este trabalho apresenta limitações, dentre as quais se destacam: o tamanho da amostra e o corte em um departamento, o que limita a possibilidade generalização de seus resultados. O que se espera é que a discussão sobre valores possa contribuir para o estabelecimento de instrumentos adequados, capazes de oferecerem uma visão dos valores dos indivíduos que atuam na área de Tecnologia de 
Informação proporcionando aos gestores e acadêmicos ferramentas para entender e analisar com profundidade como o grupo se constitui e como percebe o mundo a sua volta, quais são suas expectativas e o que valoriza como indivíduo.

\section{Referências bibliográficas:}

COUGER, J. Daniel, ZAWACKI, Robert. "Motivating and Managing Computer Personnel". John Wiley and Sons, New York, 1980.

DE SOUZA, E. G. "Cultura e Motivação dos Profissionais de Tecnologia da Informação no Brasil". Anais do VII Seme $A D$ - Seminários em Administração FEA/USP, São Paulo, SP, 2004.

DE SOUZA, E. G.; FONTANA E. R.. Estratégia Aplicada a Sistemas de Informação. $1^{\circ}$ Simpósio Brasileiro de Sistemas de Informação, PUC/RS, Porto Alegre, RS, 2004.

HOFSTEDE, Geert. Culturas e Organizações: Compreender a Nossa Programação mental. Lisboa: Edições Silabo, 2001

McNURLIN, B. C. e SPRAGUE Jr. R. H. Information Systems Management in Practice, (Fifth Edition) Prentice Hall, Upper Sadle River, 2002.

OLIVEIRA, A. F. e TAMAYO, A. Inventário de Perfis de Valores Organizacionais. RAUSP, 2004

PORTO, Juliana Barros; TAMAYO, Álvaro. Escala de valores relativos ao trabalho EVT. In XXXII Reunião Anual de Psicologia, 2003

ROHAN, Meg J. A Rose by Any Name? The Values Construct. Personality and Social Psychology Review, vol. 4, no. 3, 2000

ROKEACH, M. The nature of human values. Nova York: Free Press, 1973

ROS, Maria. Psicologia Social de los valores humanos. Madrid: Biblioteca Nueva, 2001

SCHWARTZ, S. Universals in the Content and Structure of Values: Theoretical Advances and Empirical Tests in 20 Countries. In: M.P.Zanna (Ed.) Advances in Experimental Social Psychology (Vol. 24). San Diego: Academic, 1992

SCHWARTZ, S. Value Priorities and Behavior: Applying a Theory of Integrated Value Systems. In C. Seligman, J. M. Olson, \& M. P. Zanna (Eds.), The Ontario symposium: The psychology of values (Vol. 8). Mahwah, NJ: Lawrence Erlbaum Associates Inc., 1996.

SCHWARTZ, S. A Theory of Cultural Values and Some Implications for Work. Applied Psychology: An International Review, 1999

SCHWARTZ, S. Existen Aspectos Universales en la Cultura y Contenido de los Valores Humanos? In Ros, Maria. Psicologia Social de los Valores Humanos. Madrid: Biblioteca Nueva, 2001

SCHWARTZ, S. H.; BILSKY, W. Toward a Psychological Structure of Human Values. Journal of Personality and Social Psychology, 1987

STEVENSON, W. Estatística aplicada à administração. Tradução: Alfredo Alves de Farias. São Paulo: Harper \& Row do Brasil, 1981

TAMAYO, Álvaro. Hierarquia de valores transculturais e brasileiros. Revista Quadrimestral do Instituto de Psicologia, vol 10, no.2, maio/agosto, 1994

TAMAYO, A; SCHWARTZ, S. Estrutura motivacional dos valores humanos. Psicologia: teoria e pesquisa, vol 9. no.2, maio/agosto 1993

WHITTINGTON, R. What is Strategy - and Does it Matter? International Thomson Business Press, London, 2001. 